\title{
A CHARACTERIZATION OF $M_{0}$-SPACES
}

\author{
HEIKKI J. K. JUNNILA
}

\begin{abstract}
The following result is obtained.
THEOREM. $A T_{1}$-space has a $\sigma$-closure-preserving base consisting of clopen sets iff the space can be embedded in the product of countably many $\sigma$-discrete stratifiable spaces.
\end{abstract}

1. Introduction. In [7], R. W. Heath and the author called a topological space an $M_{0}$-space if the space has a $\sigma$-closure-preserving base by clopen (= closed and open) sets. Every $M_{0}$-space is a strongly zero-dimensional $M_{1}$-space. In [13], J. Nagata asked whether every strongly zero-dimensional $M_{1}$-space is an $M_{0}$-space.

G. Gruenhage showed in [6] that every $\sigma$-discrete stratifiable space is an $M_{1}$-space and his proof actually established that such a space is an $M_{0}$-space. It is easily seen that the property of being an $M_{0}$-space is hereditary and countably productive. Consequently, every subspace of the product of countably many $\sigma$-discrete stratifiable spaces is an $M_{0}$-space; in the following we show that, among $T_{1}$-spaces, all $M_{0}$-spaces can be obtained (up to homeomorphisms) in this manner. A particular case of our result was proved in [9], where it was shown that every strongly zero-dimensional $F_{\sigma}$-metrizable stratifiable space is homeomorphic with a subspace of the product of countably many $\sigma$-discrete stratifiable spaces (a topological space is $F_{\sigma}$-metrizable if the space can be covered by countably many closed and metrizable subspaces).

Our topological terminology follows that of [5]. For basic facts concerning stratifiable and semistratifiable spaces, see [3, 1, 4 and 10]. For notation and terminology concerning neighbornets, see [8].

2. The characterization. To prove our theorem, we need the following lemma.

LEMMA. Let $(X, \tau)$ be a strongly zero-dimensional, paracompact semistratifiable Hausdorff-space, and let $V$ be a transitive neighbornet of $(X, \tau)$. Then there exists a strongly zero-dimensional metrizable topology $\pi$ on $X$ such that $\pi \subset \tau$ and $V$ is $a$ neighbornet of the space $(X, \pi)$.

PROOF. By Corollary 4.10 of [8], there exists a closed cover $\mathscr{F}=\bigcup_{n<\omega} \mathscr{F}_{n}$ of $(X, \tau)$ such that for each $n<\omega$, the family $\mathscr{F}_{n}$ is discrete and, for each $F \in \mathscr{F}, V F=V\{x\}$ for every $x \in F$. Note that, for each $n<\omega$, the family

$$
\mathscr{V}_{n}=\left\{(V F) \sim \cup\left(\mathscr{F}_{n} \sim\{F\}\right): F \in \mathscr{F}_{n}\right\} \cup\left\{X \sim \cup \mathscr{F}_{n}\right\}
$$

Received by the editors June 22, 1983.

1980 Mathematics Subject Classification. Primary 54E20. 
is an open cover of $(X, \tau)$. As a semistratifiable Hausdorff-space, $(X, \tau)$ has a $G_{\delta}$-diagonal. Consequently, there exists a sequence $\left\langle\mathscr{U}_{n}\right\rangle_{n<\omega}$ of open covers of $(X, \tau)$ such that for every $x \in X$, we have $\bigcap_{n<\omega} \operatorname{St}\left(x, \mathscr{U}_{n}\right)=\{x\}$. Since $(X, \tau)$ is a strongly zero-dimensional paracompact space, every open cover of $(X, \tau)$ has a disjoint open refinement. It follows that there exists a sequence $\left\langle\mathscr{W}_{n}\right\rangle_{n<\omega}$ of disjoint open covers of $(X, \tau)$ such that, for each $n<\omega, \mathscr{W}_{n+1}$ refines $\mathscr{W}_{n}$ and $\mathscr{W}_{n}$ refines both $\mathscr{V}_{n}$ and $\mathscr{U}_{n}$. Let $\pi$ be the topology on $X$ that has the family $\cup_{n<\omega} \mathscr{W}_{n}$ as a base. It is easily seen that $\pi \subset \tau$ and that the space $(X, \pi)$ is strongly zero-dimensional and metrizable. To show that $V$ is a neighbornet of $(X, \pi)$, let $x \in X$. Then there exists $n<\omega$ and $F \in \mathscr{F}_{n}$ such that $x \in F$. It is easy to see that the set $(V F) \sim \cup\left(\mathscr{F}_{n} \sim\{F\}\right)$ is the only member of $\mathscr{V}_{n}$ that contains $x$; it follows, since $\mathscr{W}_{n}$ refines $\mathscr{V}_{n}$, that $\operatorname{St}\left(x, \mathscr{W}_{n}\right) \subset$ $V F$. Since $V F=V\{x\}$ and since $\operatorname{St}\left(x, \mathscr{W}_{n}\right)$ is a neighborhood of $x$ in $(X, \pi)$, we have shown that $V\{x\}$ is a neighborhood of $x$ in $(X, \pi)$.

COROLlary. Let $(X, \tau)$ be a strongly zero-dimensional, paracompact semistratifiable Hausdorff-space, and let $\mathscr{F}$ be a closure-preserving family of closed subsets of $(X, \tau)$. Then there exists a strongly zero-dimensional metrizable topology $\pi$ on $X$ such that $\pi \subset \tau$ and the family $\mathscr{F}$ is closure-preserving and closed in $(X, \pi)$.

Proof. Define a transitive neighbornet $V$ of $(X, \tau)$ by setting, for each $x \in X$, $V\{x\}=X \sim \cup\left(\mathscr{F} \sim \mathscr{F}_{x}\right)$, where $\mathscr{F}_{x}=\{F \in \mathscr{F}: x \in F\}$. Let $\pi$ be a topology on $X$ that satisfies the conclusion of the lemma. Note that, for all $x \in X$ and $F \in \mathscr{F}$, if $F \cap V\{x\} \neq \varnothing$, then $x \in F$. It follows, since $V$ is a neighbornet of $(X, \pi)$, that the family $\mathscr{F}$ is closure-preserving and closed in $(X, \pi)$.

REMARK. The above results remain valid if all occurrences of the phrase "strongly zero-dimensional" are deleted; in this modified form, the result of the corollary is a slight generalization of a result obtained independently by S. Oka and Ju. H. Bregman (Lemma 3.12 of [14] and Proposition 1 of [2]).

We are now ready to prove our main result.

THEOREM. $A T_{1}$-space is an $M_{0}$-space iff the space is homeomorphic with a subspace of the product of countably many $\sigma$-discrete stratifiable spaces.

Proof. It was observed in the introduction that the condition in the theorem is sufficient for a space to be an $M_{0}$-space. To prove the necessity of the condition, let the $T_{1}$-space $(X, \tau)$ be an $M_{0}$-space. Let $\mathscr{B}=\cup_{n<\omega} \mathscr{B}_{n}$ be a base for $\tau$ such that $\mathscr{B}$ consists of clopen sets and, for each $n<\omega$, the family $\mathscr{B}_{n}$ is closure-preserving in $(X, \tau)$. Without loss of generality, we can assume that each $\mathscr{B}_{n}$ is closed under finite intersections. We show that for each $n<\omega$, there exists an $F_{\sigma}$-metrizable $M_{0}$-topology $\eta_{n}$ on $X$ such that $\mathscr{B}_{n} \subset \eta_{n} \subset \tau$. Let $n<\omega$ be given. Denote by $\mathscr{P}_{n}$ the partition of $X$ determined by the sets $\cap \mathscr{C} \sim U\left(\mathscr{B}_{n} \sim \mathscr{C}\right)$, where $\mathscr{C} \subset \mathscr{B}_{n}$ (we let $\cap \varnothing=X$ ). It follows from Theorems 3.14 and 4.8 (and Remark 4.8.2 ${ }^{\circ}$ ) of [8] that the partition $\mathscr{P}_{n}$ has a refinement $\bigcup_{k<\omega} \mathscr{F}_{n, k}$ such that each $\mathscr{F}_{n, k}$ is closed and discrete in $(X, \tau)$. Since the supremum of countably many strongly zero-dimensional metrizable topologies on $X$ is a strongly zero-dimensional metrizable topology on $X$, it follows from the above corollary that there exists a strongly zero-dimensional metrizable topology $\pi_{n}$ 
on $X$ such that $\pi_{n} \subset \tau$ and each of the families $\mathscr{B}_{n}$ and $\mathscr{F}_{n, k}, k<\omega$, is closure-preserving and closed in $\left(X, \pi_{n}\right)$. Note that for every $k<\omega$, since $\mathscr{F}_{n, k}$ is a closure-preserving and disjoint family of closed subsets of $\left(X, \pi_{n}\right), \mathscr{F}_{n, k}$ is a discrete family in $\left(X, \pi_{n}\right)$. Denote by $\eta_{n}$ the topology on $X$ that has the family $\mathscr{B}_{n} \cup \pi_{n}$ as a subbase, and note that $\mathscr{B}_{n} \subset \eta_{n} \subset \tau$. We show that $\eta_{n}$ is an $M_{0}$-topology. Since $\pi_{n}$ is a strongly zero-dimensional metrizable topology, $\pi_{n}$ has a base $\cup_{k<\omega} \mathscr{D}_{n, k}$, where each $\mathscr{D}_{n, k}$ is a discrete family of clopen subsets of $\left(X, \pi_{n}\right)$. Since the families $\mathscr{B}_{n}$ and $\mathscr{D}_{n, k}, k<\omega$, are closure-preserving and closed in $\left(X, \pi_{n}\right)$, the families $\mathscr{E}_{n, k}=\left\{B \cap D: B \in \mathscr{B}_{n}\right.$ and $\left.D \in \mathscr{D}_{n, k}\right\}, k<\omega$, are closure-preserving and closed in $\left(X, \pi_{n}\right)$ and hence also in $\left(X, \eta_{n}\right)$. Clearly, the $\sigma$-closure-preserving family $\cup_{k<\omega} \mathscr{E}_{n, k}$ is a base for the topology $\eta_{n}$. Since $\bigcup_{k<\omega} \mathscr{E}_{n, k}$ consists of clopen subsets of $\left(X, \eta_{n}\right)$, we have shown that $\left(X, \eta_{n}\right)$ is an $M_{0}$-space. Next we show that $\left(X, \eta_{n}\right)$ is $F_{\sigma}$-metrizable. For every $k<\omega$, denote by $F_{n, k}$ the set $\cup \mathscr{F}_{n, k}$; note that this set is closed in $\left(X, \pi_{n}\right)$ and hence also in $\left(X, \eta_{n}\right)$. Since $\bigcup_{k<\omega} F_{n, k}=X$, the $F_{\sigma}$-metrizability of $\left(X, \eta_{n}\right)$ follows once we show that, for each $k<\omega$, the restriction $\eta_{n, k}$ of the topology $\eta_{n}$ to $F_{n, k}$ is the same as the restriction $\pi_{n, k}$ of the metrizable topology $\pi_{n}$ to $F_{n, k}$. Since $\pi_{n} \subset \eta_{n}$, we have $\pi_{n, k} \subset \eta_{n, k}$. To show that $\eta_{n, k} \subset \pi_{n, k}$, let $x \in F_{n, k}$ and let $G$ be a neighborhood of $x$ in the topology $\eta_{n, k}$. Then there exist $B \in \mathscr{B}_{n}$ and $D \in \pi_{n}$ such that $x \in B \cap D \cap$ $F_{n, k} \subset G$. Let $F$ be that member of $\mathscr{F}_{n, k}$ which contains $x$. From the definition of $\mathscr{P}_{n}$ and $\mathscr{F}_{n, k}$, it follows that $F \subset \bigcap\left\{C \in \mathscr{B}_{n}: x \in C\right\} \subset B$; consequently, $F \cap D \subset G$. But the set $F$ is open in $\left(F_{n, k}, \pi_{n, k}\right)$ because $F \in \mathscr{F}_{n, k}$ and the family $\mathscr{F}_{n, k}$ is a discrete closed cover of $\left(\mathscr{F}_{n, k}, \pi_{n, k}\right)$. It follows from the foregoing that the set $G$ is a neighborhood of $x$ in $\left(F_{n, k}, \pi_{n, k}\right)$. We have shown that $\eta_{n, k} \subset \pi_{n, k}$; hence $\eta_{n, k}=\pi_{n, k}$ and the topology $\eta_{n, k}$ is metrizable.

Since $\mathscr{B}_{n} \subset \eta_{n} \subset \tau$ for each $n<\omega$, and since $\cup_{n<\omega} \mathscr{B}_{n}$ is a base for $\tau$, we have that $\tau=\sup _{n<\omega} \eta_{n}$. It follows that $(X, \tau)$ is homeomorphic with a subspace of the product space $\prod_{n<\omega}\left(X, \eta_{n}\right)$. By Corollary 3.3 of [9], each one of the spaces $\left(X, \eta_{n}\right)$ is homeomorphic with a subspace of the product of countably many $\sigma$-discrete stratifiable spaces. From the foregoing it follows that $(X, \tau)$ is homeomorphic with a subspace of the product of countably many $\sigma$-discrete stratifiable spaces.

REMARK. It follows from the above proof that every $M_{0^{-}}, T_{1}$-space is homeomorphic with a closed subspace of the product of countably many $F_{\sigma}$-metrizable stratifiable spaces.

In [12], K. Nagami called a topological space a $\mu$-space if the space can be embedded in the product of countably many paracompact $F_{\sigma}$-metrizable spaces. It follows from the above theorem that every $M_{0^{-}}, T_{1}$-space is a $\mu$-space. T. Mizokami has shown that every strongly zero-dimensional stratifiable $\mu$-space is an $M_{0}$-space [11, Corollary 4.7]. Consequently, we have the following result.

Proposition. $A T_{1}$-space is an $M_{0}$-space iff the space is a strongly zero-dimensional stratifiable $\mu$-space.

Added IN Proof. The result of the Proposition has been obtained independently by M. Itō [ $M_{0}$-spaces are $\mu$-spaces, preprint]. 


\section{REFERENCES}

1. C. J. R. Borges, Stratifiable spaces, Pacific J. Math. 17 (1966), 1-16.

2. Ju. H. Bregman, $A$ note about $M_{1}$-spaces and stratifiable spaces, Comment Math. Univ. Carolin. 24 (1983), 23-30.

3. J. G. Ceder, Some generalizations of metric spaces, Pacific J. Math. 11 (1961), 105-125.

4. G. D. Creede, Concerning semi-stratifiable spaces, Pacific J. Math. 32 (1970), 47-54.

5. R. Engelking, General topology, PWN, Warszawa, 1977.

6. G. Gruenhage, Stratifiable o-discrete spaces are $M_{1}$, Proc. Amer. Math. Soc. 72 (1978), 189-190.

7. R. W. Heath and H. J. K. Junnila, Stratifiable spaces as subspaces and continuous images of $M_{1}$-spaces, Proc. Amer. Math. Soc. 83 (1981), 146-148.

8. H. J. K. Junnila, Neighbornets, Pacific J. Math. 76 (1978), 83-108.

9. On strongly zero-dimensional $F_{\mathrm{\sigma}}$-metrizable stratifiable spaces, (Proc. Leningrad Internat. Topological Conf., 1982), Lecture Notes in Math., Springer-Verlag, Berlin (to appear).

10. J. A. Kofner, On pseudostratifiable spaces, Fund. Math. 70 (1971), 25-47. (Russian)

11. T. Mizokami, On M-structures, Topology Appl. 17 (1984), 63-89.

12. K. Nagami, Normality of products, Actes, Congres Intern. Math., vol. 2, Gauthier-Villars, Paris, 1971, pp. 33-37.

13. J. Nagata, On Hyman's M-space (R. Dickman and P. Fletcher, eds.), Topology Conf. (Blacksburg, Virginia, 1973), Lecture Notes in Math., Vol. 375, Springer-Verlag, Berlin, 1974, pp. 198-208.

14. S. Oka, Dimension of stratifiable spaces, Trans. Amer. Math. Soc. 275 (1983), 231-243.

Department of Mathematics, University of Helsinki, Hallituskatu 15, 00100 Helsinki 10, FINLAND 